sich beim Schütteln mit Luft zu leicht oxydirt und diese Oxydation irrthümlich als Wirkung des Chlors in Rechnung kommt.

4) Die Jodkaliumprobe von Schwarz ist zuverlässig beim Chlorwasser, giebt aber zu hohe Werthe beim Chlorkalk.

5) Die Exhalation des Chlorwassers aus einer zu mehren vergleichenden Versuchen bestimmten Flasche ist bei deren successiver Entleerung so bedeutend, dass dies auch für die kürzesten Zeitfristen beachtet werden muss.

\title{
Ueber das chromsaure Chromoxyd.
}

\section{Von 0. Popp. *)}

Wenn eine Lösung von unterschwefligsaurem Natron mit einer Lösung von saurem chromsauren Kali erhitzt wird, so bildet sich ein brauner Niederschlag von chromsaurem Chromoxyd. Wird die Lösung des $\mathrm{NaO}, \mathrm{S}^{2} \mathrm{O}^{2}$ mit der Kalibichromatlösung in der Kälte vermischt, so findet momentan scheinbar keine Einwirkung statt; nach kurzer Zeit bilden sich braune wolkige Ablagerungen, die Menge des Niederschlages wird allmählig stärker und ist nach einigen Tagen fast vollständig.

Bringt man die Lösung des Natronhyposulfits erst zum Kochen und fügt dann allmählig die Kalibichromatlösung hinzu, so findet die Ausscheidung des braunen Niederschlages sofort statt; doch bleibt auch hier immer ein kleiner Theil gelöst, der sich erst nach einiger Zeit vollständig niederschlägt. In beiden Fällen ist der copiöse braune Niederschlag hydratisches chromsaures Chromoxyd. Die nach der Zersetzung resultirende Lösung enthält, ausser etwas unzersetztem Natronhyposulfit, neutrales Kalichromat, schwefelsaures und schwefiigsaures Salz. Die Reaction kann durch folgende Zersetzungsgleichung wiedergegeben werden:

$$
\begin{aligned}
2\left(\mathrm{KO}, 2 \mathrm{CrO}^{3}\right) & +\mathrm{NaO}, \mathrm{S}^{2} \mathrm{O}^{2}=\mathrm{KO}, \mathrm{CrO}^{3}+\mathrm{Cr}^{2} \mathrm{O}^{3}, \mathrm{CrO}^{3} \\
& +\mathrm{KO}, \mathrm{SO}^{3}+\mathrm{NaO}, \mathrm{SO}^{2} .
\end{aligned}
$$

*) Als Separatabdruck aus den Annalen d. Chem. u. Pharmacie. Bd. 156 (1870) rom Hrn. Verfasser eingesandt.

H. $L$. 
Es ist jedoch sehr wahrscheinlich, dass primitiv der Bildung der schwefligen und Schwefelsäure die der Unterschwefelsäure vorausgeht und erstere beiden die Zersetzungsproducte der letzteren sind. Der Reactionsgleichung würde man demnach folgenden Ausdruck geben müssen:

$$
\begin{gathered}
2\left(\mathrm{KO}, 2 \mathrm{CrO}^{3}\right)+\mathrm{NaO}, \mathrm{S}^{2} \mathrm{O}^{2}=\mathrm{KO}, \mathrm{CrO}^{3}+\mathrm{Cr}^{2} \mathrm{O}^{3}, \mathrm{CrO}^{3} \\
+\mathrm{NaO}, \mathrm{S}^{2} \mathrm{O}^{5}+\mathrm{KO},
\end{gathered}
$$

welch' letzteres, besonders in der Hitze, die Bildung der schwefligen und Schwefelsäure veranlassen und unterstützen würde.

Das Auftreten des' chromsauren Chromoxyds von der angenommenen Formel $\mathrm{Cr}^{2} \mathrm{O}^{3}, \mathrm{CrO}^{3}$ kann als normales und primitives aufgefasst werden; doch ist dessen Bildung an ganz bestimmte Bedingungen geknüpft. Dasselbe bildet sich nur dann, wenn die Zersetzung in der Kälte geschieht und die Verbindung nicht zu lange ausgewaschén wird. Wird die Verbindung in der Hitze gebildet und das Answaschen so lange fortgesetzt, bis das ablaufende Waschwasser nicht mehr gelb gefärbt erscheint, so resultirt stets eine Verbindung, welche der Formel $2 \mathrm{Cr}^{2} \mathrm{O}^{3}, \mathrm{CrO}^{3}$ entspricht, und ist dieses Verhalten auf die Eigenschaft des einfach-basischen Salzes, in ein überbasisches überzugehen, begründet.

Das so dargestellte chromsaure Chromoxyd ist sehr hydratisch und, bei gelinder Wärme getrocknet, ein leichtes voluminöses Pulver von tief gelbbrauner Farbe. Es löst sich, besonders beim Erwärmen, leicht in verdünnter Schwefelund Salzsäure auf mit intensiv grüner Farbe; in verdünnter Essigsäure ist es schwerer löslich. Salpetersäure löst es mit tief brauner Farbe, concentrirte Salzsäure beim Erwärmen unter Chlorentwickelung auf. Aus den Aufösungen in verdünnter Salz- und Schwefelsäure wird dorch Ammoniak grünes Chromoxyd gefällt; in Lösung bleibt Ammonchromat, gewöhnlich etwas Chromoxyd gelöst enthaltend.

Wird die Verbindung im Platintiegel vorsichtig erhitzt, so entweicht zuerst das Hydratwasser; bei gesteigertem Erhitzen tritt ein heftiges Glühphänomen ein; es wird Sauerstoff entwickelt und reines Chromoxyd von schön grüner

Aroh, d. Pharm. OXCVh. Bds. 2. Hn. 
Farbe bleibt zurück. Mit kohlensauren und aetz. Alkalien gekocht wird grünes Chromoxyd ausgeschieden und Chromat gebildet.

Die Analyse der Verbindungen geschah in der Weise, dass dieselben mit Natroncarbonat so lange gekocht wurden, bis das gebildete Chromoxyd rein grün war, und dieses als solches direct bestimmt. Das in Lösung gegangene Chromat wurde durch schweflige Säure reducirt und als Chromoxyd bestimmt.

Wie schon oben erwähnt, war es nur möglich eine Verbindung zu erhalten, welche der Formel $\mathrm{Cr}^{2} \mathrm{O}^{3}, \mathrm{CrO}^{3}$ entsprach, wenn die Zersetzung in der Kälte geschah und das Auswaschen nicht zu lange fortgesetzt wurde; gewöhnlich resultirten Verbindungen, welche den Formeln $\mathrm{Cr}^{2} \mathrm{O}^{3}, \mathrm{CrO}^{3}$ and $2 \mathrm{Cr}^{2} \mathrm{O}^{3}, \mathrm{CrO}^{3}$ intermediär waren. Geschah die Bildung der Verbindung in der Siedhitze und wurde das Auswaschen derselben so lange fortgesetzt, bis das Waschwasser farblos erschien, so entsprach die Zusammensetzung der Verbindung immer der Formel $2 \mathrm{Cr}^{2} \mathrm{O}^{3}, \mathrm{CrO}^{3}$, und zwar mit 9 Aequivalenten Hydratwasser, so dass die Formel des hydratischen chromsauren Chromoxyds als $2 \mathrm{Cr}^{2} \mathrm{O}^{3}, \mathrm{CrO}^{3}+9$ aq. aufgefasst werden kann.

Dieses überbasische chromsaure Chromoxyd scheint die beständigste der eventuellen Verbindungsgrade zu sein, welche das Chromoxyd mit der Chromsäure eingehen kann.

Laboratorium in Göttingen, den 15. Juli 1870.

\section{Ueber Persea gratissima Gaertner (Laurineae).}

Von Dr. Theodor Peckolt in Rio de Janeiro.

Abacateira, Avacatenbaum, Abacatenbirne (Pereira Abacate), Loureiro Abacate, Abacatenlorbeer. Ist in einigen Werken Advogatobaum benannt, welches man von Advocaten herrührend glauben könnte; doch ist der ursprüngliche von den Caraiben herstammende Name 